\title{
Simulation mit Supercomputern Ein neues Werkzeug der Physik
}

\author{
H. Ruder, T. Ertl, F. Geyer, H. Herold, U. Kraus, H.-P. Nollert, A. Rebetzky, \\ W. Schweizer, C. Zahn \\ Lehr- und Forschungsbereich Theoretische Astrophysik der Universität Tübingen
}

\section{Zusammenfassung und Einleitung}

Unser Wissen über die Struktur des Kosmos und die darin enthaltenen Objekte stammt aus der sorgfältigen Analyse der auf der Erde einfallenden elektromagnetischen Strahlung, verbunden mit einer theoretischen Modellierung im Rahmen der von uns erforschten Naturgesetze. Die astronomischen Beobachtungen erstrecken sich dabei heute vom Radiowellenbereich über den Infrarot-, den optischen, den Röntgenbereich bis hin zum HöchstenergieGamma-Bereich, also über mehr als 20 Dekaden des elektromagnetischen Spektrums.

Die sprunghafte Zunahme unserer Kenntnisse in den letzten zwei Jahrzehnten verdanken wir vor allem den vielen Forschungssatelliten, die die Beobachtung kosmischer Objekte ohne die störenden Einflüsse unserer Atmosphäre erlauben. Speziell im weichen Röntgenbereich ist dadurch eine Beobachtung überhaupt erst möglich, und es ist gerade dieser Spektralbereich, der uns wesentlich neue Erkenntnisse über hochinteressante Objekte geliefert hat, da intensive Röntgenstrahlung nur unter sehr extremen physikalischen Bedingungen entsteht.

Die in diesen Röntgenemissionsgebieten vorherrschenden Temperaturen, Magnet-, und Gravitationsfelder sind so extrem, daß sie in irdischen Labors nicht realisiert werden können. Folglich bleibt nur die Möglichkeit, die Eigenschaften der Materie und die unter diesen Bedingungen ablaufenden physikalischen Prozesse theoretisch zu berechnen, um so - im Vergleich mit den Beobachtungen - zu zuverlässigen Aussagen über die Struktur dieser kosmischen Objekte zu gelangen. Die möglichst realistische Computersimulation der Systeme im Rahmen einer beobachtungsnahen Theorie erfordert vor allem bei Parameterstudien im allgemeinen den Einsatz der größten verfügbaren Rechenleistungen.

Die theoretische Modellierung überdeckt heute ein breites Spektrum an astrophysikalischen Fragestellungen, wie z.B. die Dynamik des Planetensystems, die Entstehung protoplanetarischer Scheiben und Magnetosphären, Sternentwicklung, Supernovaexplosionen, Sternkollisionen, Akkretionsscheiben, Akkretionssäulen, Galaxienentwicklung, Jets usw. Sie führt auf die gekoppelten Differentialgleichungssysteme der Hydrodynamik, der Magnetohydrodynamik, des Strahlungstransports und der komplexen Vielteilchensysteme. Diese wiederum werden mit Codes für elliptische und hyperbolische Gleichungen, mit Teilchensimulationsund Monte-Carlo-Codes, sowie mit Verfahren zur hochgenauen Integration numerisch gelöst. Eine exemplarische, keineswegs vollständige Zusammenstellung verschiedener astrophysikalischer Objekte und der zu ihrer Simulation angewandten Methoden findet sich in Tab. 1.1. 
Tab. 1.1: Beispiele für Simulationsmethoden und ihre Anwendung auf astrophysikalische Objekte

\begin{tabular}{|c|c|c|c|}
\hline $\begin{array}{l}\text { Numerische Lösung der } \\
\text { Bewegungsgleichungen von } \\
\text { Vielteilchensystemen }\end{array}$ & $\begin{array}{l}\text { Teilchensimulation } \\
\text { (Particle-In-Cell) }\end{array}$ & $\begin{array}{l}\text { Hydrodynamische und } \\
\text { magnetohydrodynami- } \\
\text { sche Simulationen }\end{array}$ & Monte-Carlo-M \\
\hline $\begin{array}{l}\text { Dynamik des Planeten- } \\
\text { systems }\end{array}$ & $\begin{array}{l}\text { Protoplanetare } \\
\text { Scheiben }\end{array}$ & Akkretionsphänomene & Kosm \\
\hline Dynamik von Sternhaufen & Akkretionsscheiben & $\begin{array}{l}\text { Überschallströmun- } \\
\text { gen, Jets }\end{array}$ & $\begin{array}{l}\text { Gamma-Ray-Burster } \\
\text { Photonenausbreitung }\end{array}$ \\
\hline Galaxienentwicklung & Magnetosphären & Scheiben & \\
\hline Galaxienkollisionen & Sternwinde & Interstellares Medium & \\
\hline
\end{tabular}

Im Folgenden soll an drei Beispielen etwas genauer beschrieben werden, wie bei Magnetfeldern von $10^{8}$ Tesla, Temperaturen von einigen 100 Millionen Grad und Gravitationsfeldern mit der $10^{12}$-fachen Stärke des Erdfeldes, also bei Bedingungen, die unsere experimentellen Möglichkeiten um viele Größenordnungen überschreiten, durch Computersimulationen detaillierte Aussagen über komplexe physikalische Vorgänge erzielt werden können

\section{Eigenschaften der Materie in starken Magnetfeldern}

In diesem Abschnitt wollen wir uns mit der Physik in extrem starken Magnetfeldern beschäftigen. Diese riesigen Magnetfelder treten bei kompakten kosmischen Objekten, nämlich den Weißen Zwergsternen und den Neutronensternen auf. Beide sind Endstadien der Sternentwicklung, bei denen im Inneren keine Energieerzeugung mehr stattfindet und die Materie daher so weit zusammengedrückt ist, daß sıe allein ohne Strahlungsdruck die Gravitationskraft kompensieren kann. Die Größe der Magnetfelder läßt sich dann einfach dadurch erklären, daß beı dem Gravitationskollaps wegen der hohen elektrischen Leitfähıgkeit der Sternmaterie der magnetische Fluß erhalten bleibt und somit das Magnetfeld umgekehrt proportional zur Querschnittsfläche anwächst. Mit den in Tab. 21 zusammengestellten typischen Werten erkennt man, daß Ausgangsfeldstärken von $10^{-3}-10^{-1}$ Tesla, wie man sie be Sternen durchaus mißt, genügen, um das Auftreten dieser riesigen Felder zwanglos zu erklären.

Tab. 2.1: Physikalische Parameter von Weißen Zwergsternen und Neutronensternen im Vergleich mit unserer Sonne

\begin{tabular}{lccc}
\hline & Sonne & Weiße Zwergsterne & Neutronensterne \\
\hline Masse & $2 \times 10^{30} \mathrm{~kg}=m_{\odot}$ & $\sim 1 m_{\odot}$ & $\sim(1-2) m_{\odot}$ \\
Radius & $7 \times 10^{5} \mathrm{~km}=R_{\odot}$ & $\sim 10^{4} \mathrm{~km} \approx 10^{-2} R_{\odot}$ & $\sim 10 \mathrm{~km} \approx 10^{-5} R_{\odot}$ \\
Mittlere Dichte & $1.4 \mathrm{~g} / \mathrm{cm}^{3}$ & $\sim 10^{6} \mathrm{~g} / \mathrm{cm}^{3}$ & $\leq 10^{15} \mathrm{~g} / \mathrm{cm}^{3}$ \\
Rotationsperiode & $27 \mathrm{~d}$ & $100 \mathrm{~s}-$ Tage? & $10^{-3}-10^{3} \mathrm{~s}$ \\
Magnetfeldstärke & $\sim 10^{-4}-10^{-3} \mathrm{~T}$ & $\sim 10^{2}-10^{5} \mathrm{~T}$ & $\sim 10^{7}-10^{9} \mathrm{~T}$ \\
\hline
\end{tabular}


Magnetfelder von $10^{8}-10^{9}$ Tesla sind 10 Millionen mal größer als die stärksten Felder, die man in irdischen Labors herstellen kann. Unabhängig davon, daB man sie auch praktisch nicht finanzieren könnte - ein Kubikzentimeter Feld enthält soviel Energie, daß seine Erzeugung bei den heutigen Energiepreisen etwa 10 Milliarden Mark kosten würde - sind die Lorentz-Kräfte bei diesen Feldstärken so gewaltig, daß in diesen Feldern irdische Labors zu näherungsweise eindimensionalen Schläuchen zusammenschnurren würden. Die Energiedichte solcher Felder ist tausendfach größer als die Ruhenergiedichte von Wasser. Während normale irdische Materie daher völlig von diesen Feldern dominiert wird, bedeuten sie für Neutronensternmaterie mit einer Dichte von 500 Millionen Tonnen pro Kubikzentimeter dagegen nur eine winzige Störung. Für eine korrekte Modellierung der physikalischen Prozesse in der Umgebung dieser stark magnetisierten kompakten Objekte ist aber die genaue Kenntnis des Verhaltens der Materie eine unabdingbare Voraussetzung, und die einzige Möglichkeit, diese benötigten Daten zu gewinnen, sind Computersimulationen, bei denen man ja gefahrlos das Magnetfeld beliebig groß werden lassen kann. Problematisch sind hier nicht die Kräfte sondern die CPU-Leistung und die Größe des Speichers, denn es hat sich gezeigt, daß bereits die Berechnung des einfachsten atomaren Systems, des Wasserstoffatoms, an die Grenzen einer Cray 2 stößt. Wegen der Zylindersymmetrie des Magnetfelds ist das Problem nicht mehr separierbar und daher numerisch zweidimensional zu behandeln. Um einen Eindruck von der Komplexität zu vermitteln, ist in Abb. 2.1 das Wasserstoffspektrum in Abhängigkeit vom Magnetfeld gezeigt. Die im feldfreien Fall entarteten Linien der Lyman-, Balmer-, Paschen- usw. Serien spalten bei kleinen Feldstärken in ihre ZeemanKomponenten auf, bei Feldstärken, bei denen die Lorentz- und Coulomb-Kräfte vergleichbar sind, wird das Spektrum völlig zerfleddert und erst bei sehr großen Feldstärken erkennt man wieder eine gewisse Ordnung, die aber jetzt vom Magnetfeld erzeugt wird.

Um sich eine Vorstellung machen zu können, was den Atomen in diesen Magnetfeldern passiert, muB man die Struktur der Wellenfunktionen $\Psi(x, y, z)$ analysieren. Üblicherweise berechnet man dazu Höhenlinien von $|\Psi|^{2}$, der Aufenthaltswahrscheinlichkeit des Elektrons In verschiedenen zueinander senkrechten Schnittebenen. Ein wesentlich anschaulicherer Eindruck läßt sich jedoch mit der folgenden Methode gewinnen: Man nimmt dazu an, daß das dreidimensionale skalare Feld $|\Psi|^{2}$ der Elektronendichte "Licht" emittiert, dessen Intensität proportional zu der lokal vorhandenen Elektronendichte ist und das ohne Absorption zum Beobachter gelangt. Die Elektronenwolke des Atoms stellt sich somit als ein optisch dünner selbstleuchtender Gasnebel dar. Die Intensität, die ein entfernter Beobachter aus einer bestimmten Richtung sieht, ist einfach das Integral des in seine Richtung emittierten Lichts längs des Sehstrahls. Indem man nun den Sehstrahl in einem feinen Gitter über das gesamte Atom bewegt, kann man ein Rasterbild erzeugen, das in guter Näherung das wiedergibt, was eın Beobachter tatsächlich sehen würde. Diese Bilder lassen sich natürlich für jede Beobachtungsrichtung berechnen und vermitteln einen realistischen Eindruck von der atomaren Struktur. Eine besonders gute räumliche Vorstellung gewinnt man mit Filmsequenzen, bei denen man die Atome rotieren läßt. Die Methode der Sehstrahlintegration ist zugegebenermaßen relativ rechenintensiv - für ein Bild mit einer Auflösung von $800 \times 800$ Pixel müssen 640000 eindimensionale Integrale berechnet werden - die Methode ist aber gut vektorisierbar und, trivialerweise, ideal parallelisierbar. Als ein Beispiel ist in der Abb. 2.2 die Wellenfunktion des Wasserstoffatoms für einen Zustand mit der (feldfreien) Hauptquantenzahl $n=5$ für fünf verschiedene Magnetfeldstärken dargestellt. Man erkennt an diesen Bildern deutlich, wie im Magnetfeld durch die immer stärker werdenden Lorentz-Kräfte das 


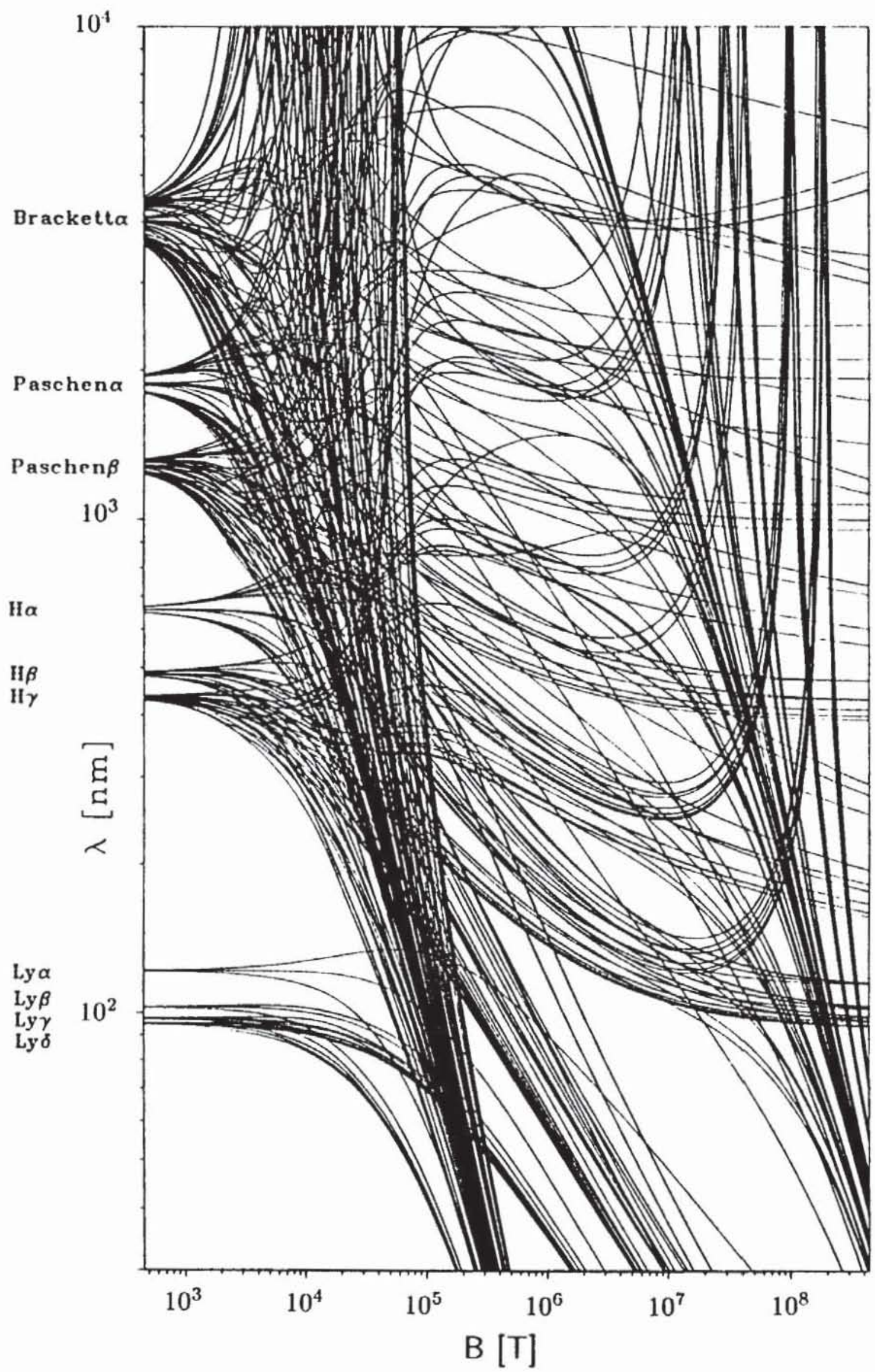

Abb. 2.1: Das Spektrum des Wasserstoffatoms in Abhängigkeit vom Magnetfeld 


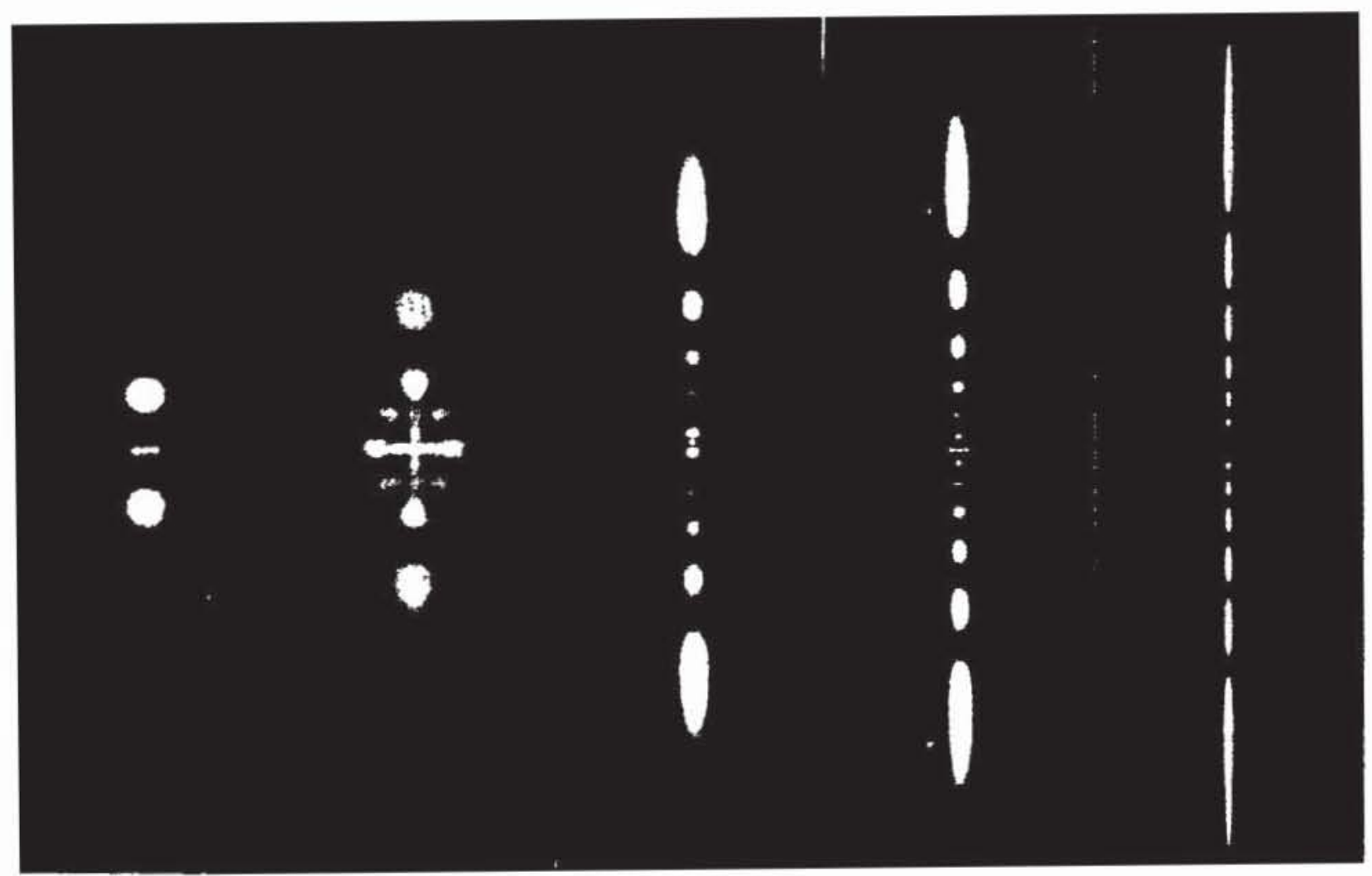

Abb. 2.2: Die Wellenfunktion des Wasserstoffatoms für einen Zustand mit der (feldfreien) Hauptquantenzahl $n=5$ für fünf verschiedene Magnetfeldstärken (von links nach rechts $B=0,5 \times 10^{3}, 2.5 \times 10^{4}$, $5 \times 10^{4}$ und $5 \times 10^{5}$ Tesla. Man erkennt deutlich, wie im Magnetfeld durch die immer stärker werdenden Lorentz-Kräfte das Atom senkrecht zum Magnetfeld zusammengeschnürt und dadurch gleichzeitig in Magnetfeldrichtung länger wird.

Atom senkrecht zum Magnetfeld zusammengeschnürt und dadurch gleichzẹitig in Magnetfeldrichtung länger wird.

Erwahnenswert ist noch, daß diese Computersimulation die einzige Möglichkeit ist, räumlich hochaufgelöste Bilder von atomaren Strukturen zu gewinnen. Die Mikroskopie von realen Atomen scheitert bei dieser Auflösung grundsätzlıch an der Heisenbergschen Lnschärferelatıon. die zwar auch fur den Computer aber nicht für die Simulation gültig ist.

Die Bedeutung einer guten Visualisierung kommt insbesondere bei komplexeren Strukturen voll zum Tragen. Als ein Beispiel seien hier hochangeregte Rydbergzustände des Wasserstoffatoms in Labormagnetfeldern genannt In diesen Zuständen mit Hauptquantenzahlen zwischen 50 und 100 wird aus dem Wasserstoffatom ein zartes und bizarres Gebilde voll künstlerischer Ästhetik mit Bindungsenergien von wenigen Millielektronenvolt und räumlichen Ausdehnungen in Magnetfeldrichtung bis zu einem tausendstel Millimeter, also schon größer als Viren. Diese Gebilde sind interessante Studienobjekte für den Übergang klassische Physik $\rightarrow$ Quantenphysik und von fundamentaler Bedeutung für Fragen im $\mathrm{Zu}$ sammenhang mit der Suche nach dem Quantenchaos. Das klassische System zeigt in diesen 

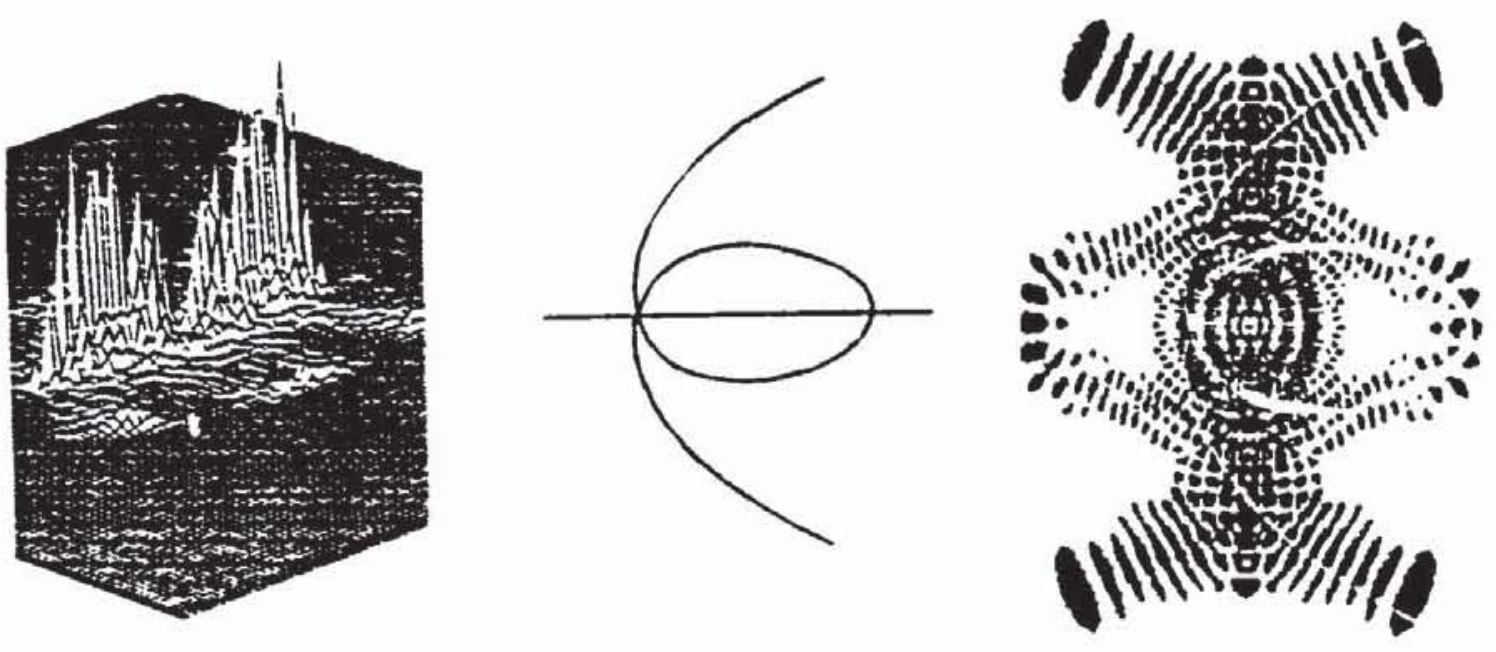

Abb. 2.3: Visualisierung einer Wellenfunktionslokalisierung
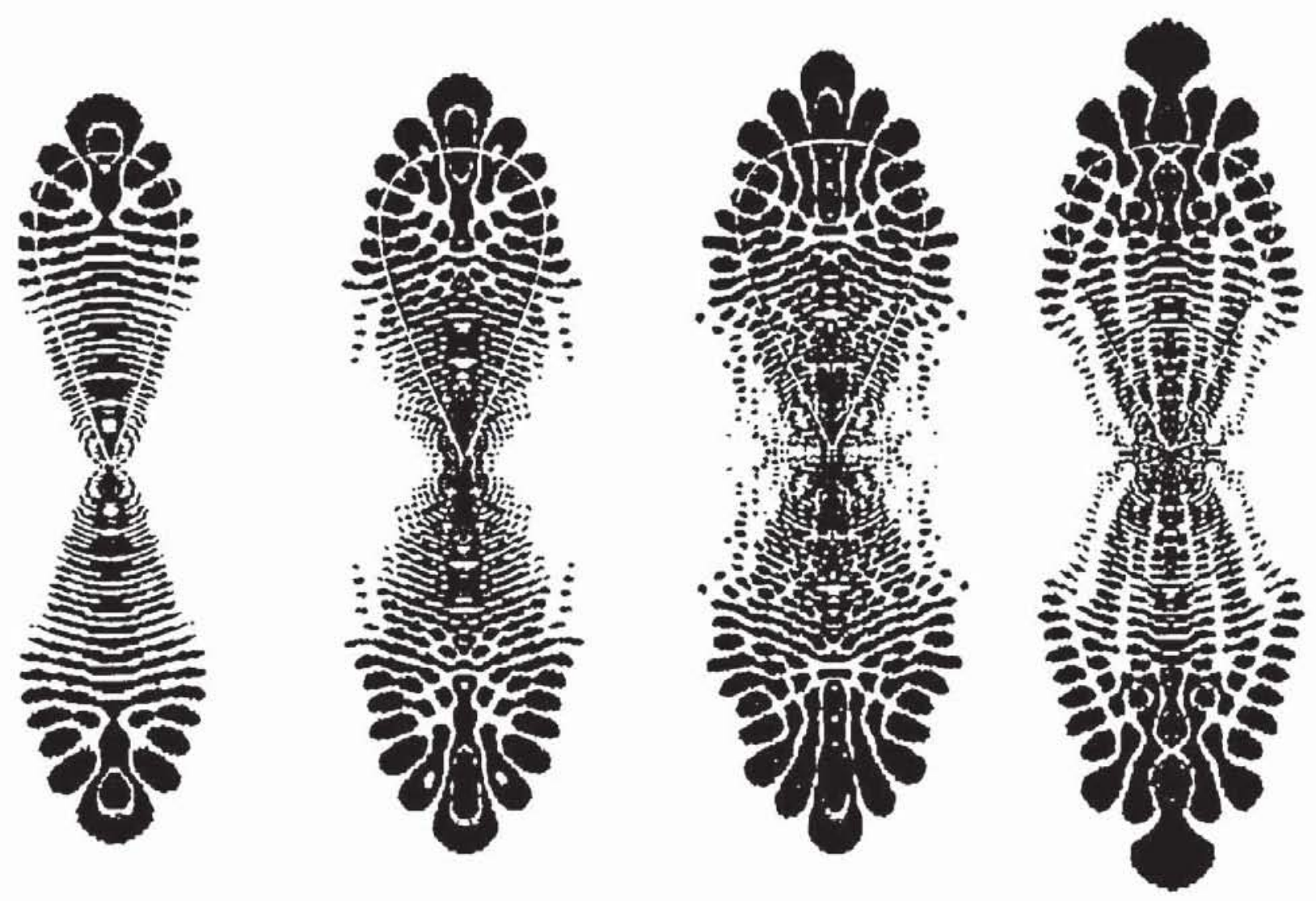

Abb. 2.4: Rydberg-Zustände des Wasserstoffatoms im starken Magnetfeld mit superponierten klassischen Bahnen

interessierenden Magnetfeld- und Energiebereichen einen Übergang von regulärem zu chaotischem Verhalten, klassische Trajektorien werden mithin zunehmend instabil. Semiklassische Vorhersagen lassen eine Lokalisierung der Elektronenbahnen, d.h. eine Überhöhung der Auf- 
enthaltswahrscheinlichkeit längs klassischer Bahnen erwarten. Mit zunehmender Chaotizität des Wasserstoffatoms im äußeren Magnetfeld sollte diese als Vernarbung der Wellenfunktion bezeichnete Eigenschaft zunehmend durch die komplexere Struktur der Wellenfunktion verwischt werden. Ein einfaches numerisches Maß, mit dem sich die Vernarbung einer strukturreichen Wellenfunktion längs einer klassisch chaotischen Bahn berechnen ließe, gibt es nicht. Die Natur hat uns Menschen jedoch mit einem der effizientesten Detektoren zum Aufspüren räumlicher Strukturen ausgerüstet: dem Auge. Verbleibt noch die wesentliche Aufgabe, die quantenmechanischen Strukturen diesem Detektor zugänglich zumachen, d.h. Lokalisierungen zu visualisieren. In Abb. 2.3 zeigen wir das typische Vorgehen: zunächst berechnen wir die Aufenthaltswahrscheinlichkeit des Wasserstoffelektrons im Magnetfeld sowie bei derselben Energie und Magnetfeldstärke klassische Trajektorien, beides dargestellt anhand der beiden linken Bilder. Durch Farbkodierung der Aufenthaltswahrscheinlichkeit und Überlagerung der klassischen Bahn erhalten wir das rechte Bild (hier eine Graukopie), das uns nun zum Detektieren von Wellenfunktionslokalisierungen dient. In diesem Beispiel befinden wir uns klassisch im Übergangsbereich von Regularität zu Irregularität, d.h. einzelne Bahnen sind noch stabil, wie die hier gezeigte, während andere Bahnen bereits instabil sind. Die nächste Abbildung 2.4 zeigt genau einen solchen Übergang auf. Bei den ersten drei Bildern von links sind die klassischen Trajektorien noch stabil, während im vierten Fall die superponierte klassische Bahn instabil ist.

\section{Kosmische Röntgenquellen}

Im Jahre 1971 wurden von dem mit hoher zeitlicher Aufösung beobachtenden Röntgensatelliten UHURU periodische Röntgenpulse registriert. Aus den Pulsperioden, die im Sekundenbereich liegen und ungeheuer konstant sind, folgt zwingend, daß es sich bei diesen kosmischen Röntgenquellen nur um rotierende Neutronensterne handeln kann. Seit ihrer Entdeckung sind diese Röntgenpulsare Gegenstand intensiver astrophysikalischer Forschungen, da sie die stärksten Röntgenquellen unserer Milchstraße sind. Die Abstrahlung eines typischen Röntgenpulsars beträgt $10^{30}$ Watt, er emittiert damit allein im Röntgenbereich das zweitausendfache der gesamten Strahlungsleistung unserer Sonne. Der Röntgenpulsar selbst ist ein stark magnetisierter rasch rotierender Neutronenstern, der zusammen mit einem normalen Stern ein enges Doppelsternsystem bildet (Abb. 3.1a). Durch seinen kleinen Radius von nur etwa $10 \mathrm{~km}$ und seiner im Vergleich dazu großen Masse von ungefähr einer Sonnenmasse ist ein Neutronenstern ein äußerst kompaktes Objekt mit einer Dichte von 500 Millionen Tonnen pro Kubikzentimeter, also dem Mehrfachen der Dichte von Atomkernen. Aufgrund seiner starken Gravitationswirkung wird Masse aus dem normalen Begleitstern herausgezogen, die auf den Neutronenstern zuströmt und um ihn herum eine dünne Scheibe bildet, die sogenannte Akkretionsscheibe (Abb. 3.1b), von deren innerem Rand die Materie dann endgültig auf die Neutronensternoberfläche stürzt. Die ionisierte Materie, die aus der Scheibe in das Magnetfeld des Neutronensterns eintritt, wird von diesem Feld zu den Magnetpolen hin gebündelt (Abb. 3.1d). Dort treffen dann bei Magnetfeldstärken von $10^{8}-10^{9}$ Tesla auf eine Fläche von nur wenigen Quadratkilometern 100 Milliarden Tonnen Materie pro Sekunde mit einer Geschwindigkeit von ca. $160000 \mathrm{~km} / \mathrm{s}$, also mit mehr als halber 
Lichtgeschwindigkeit, auf. Dabei wird die Oberfläche durch die bei der Abbremsung freigesetzte kinetische Energie auf Temperaturen von ca. 100 Millionen Grad aufgeheizt, was zur Emission der Röntgenstrahlung führt.
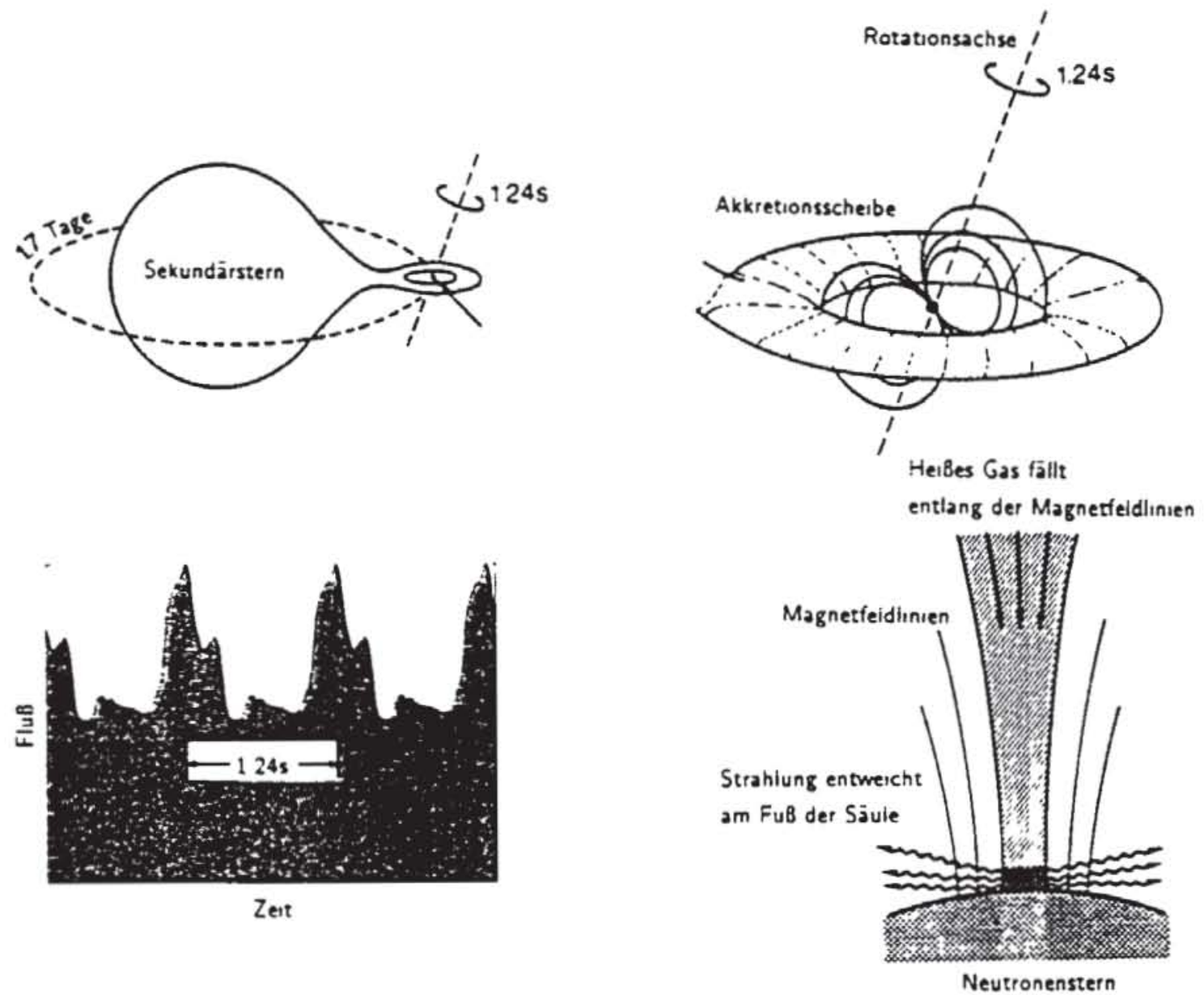

Abb. 3.1: Schematısche Darstellung des engen Röntgen-Doppelsternsystems Her X-1 im Sternbild Herkules (a). Die Matene strömt vom Sekundärstern zum Neutronenstern und bildet dabei eine Scheibe (b). Durch die schnelle Rotation des Neutronensterns sieht man die an den magnetischen Polen (d) entstehende Röntgenstrahlung gepulst (c).

Diese "kosmische Röntgenröhre", deren Abstrahlung auf eine oder mehrere Vorzugsrichtungen konzentriert ist, ist so intensiv, daß man damit einen Menschen noch in einer Entfernung von einer Bıllion Kilometern in Sekundenbruchtellen durchleuchten könnte. Da die Achse durch die magnetischen Pole im allgemeinen nicht mit der Rotationsachse zusammenfällt, sehen wir somit - wie bei einem Leuchtturm - die Röntgenstrahlung mit der Rotationsperiode gepulst (Abb. 3.1c).

Es wäre natürlich ein völlig aussichtsloses Unterfangen, zu versuchen, das gesamte Doppelsternsystem mit allen Einzelheiten bıs hin zur spektral aufgelösten Röntgenemission in einem Stück zu simulieren. Der einzig gangbare Weg ist die Zerlegung in Untersysteme 
mit wohldefinierten Schnittstellen und Wechselwirkungen. In unserem Beispiel sieht diese natürlich durch die physikalischen Gegebenheiten vorgegebene Zerlegung wie folgt aus:

- Modellierung des Sekundärsterns unter Berücksichtigung der Röntgenstrahlung und der Gezeitenwirkung des Neutronensterns,

- Berechnung der Materieüberströmrate am inneren Lagrange-Punkt,

- Simulatıon der sich um den Neutronenstern bildenden Akkretionsscheibe in Abhängigkeit von der Überströmrate,

- Stabilitätsanalysen für diese Scheiben, Berechnung ihrer Emission,

- Untersuchung der Wechselwirkungsprozesse am inneren Rand der Scheibe mit der Magnetosphäre des Neutronensterns,

- selbstkonsistente Modellierung der Akkretionssäule auf dem Neutronenstern bei vorgegebener Akkretionsrate und Magnetfeld,

- selbstkonsistente Modellierung der Abbremsregion (Hot Spot) am Fuße der Akkretionssäule auf der Neutronensternoberfläche,

- spektral und zeitlich aufgelöste Berechnung des Strahlungsflusses zum Beobachter unter Berücksichtigung der Lichtablenkung im starken Gravitationsfeld des Neutronensterns.

Zur Simulation dieser verschiedenen Teilsysteme sind jeweils ganz unterschiedliche Verfahren erforderlich. Jeder einzelne Punkt ist bereits ein sehr aufwendiges numerisches Problem. Wir wollen in diesem Abschnitt einige Ergebnisse unserer Simulationsrechnungen zur Entstehung von Akkretionsscheiben und zur Modellierung des Emissionsgebiets auf dem Neutronenstern vorstellen.

Zur Modellierung von Akkretionsscheiben sind Teilchensimulationsmethoden besonders gut geeignet. Ein "Teilchen" repräsentiert dabei ein kleines, aber makroskopisches Volumenelement, gefüllt mit Akkretionsscheibenmaterial. Die Bewegung dieser Pseudoteilchen wird durch die Newtonsche Bewegungsgleichung bestimmt. Sie enthält die Gravitationswirkung der beiden Sterne, die Zentrifugal- und die Corioliskraft. Die zwei Komponenten des Systems werden als Massenpunkte angenommen, die auf Kreisbahnen um den gemeinsamen Schwerpunkt umlaufen. Im mitrotierenden System bewegen sich die Teilchen dann unter dem Einfluß des Roche-Potentials.

In einem ersten Schritt wird mit einem schnellen Verfahren die Bewegungsgleichung numerisch integriert. Die Abb. 3.2 zeigt drei Bahnen von Einzelteilchen in diesem RochePotential, die unter leicht verschiedenen Anfangsbedingungen am inneren Lagrange-Punkt gestartet sind. Für eine realistische Simulation müssen zusätzlich der Druckgradient und die Viskosität berücksichtigt werden. Diese makroskopischen Größen müssen auf eine effektive Wechselwirkung zwischen den Gaszellen umgerechnet werden. Die viskose Wechselwirkung verursacht lokal eine Geschwindigkeitsangleichung der einzelnen Teilchen, wodurch ihre Bahnen näherungsweise kreisförmig verlaufen, und führt auf einen nach innen gerichteten Massentransport.

Mit den Beobachtungen direkt vergleichbar sind nur Lichtkurven und Spektren. Um aus den simulierten Scheiben realistische Lichtkurven zu erhalten, muß man den Strahlungstransport in der Scheibe berücksichtigen, d.h. man muß berechnen, wie die durch die viskose Wechselwirkung lokal in der Scheibe erzeugte Energie abgestrahlt wird. Außerdem hängt die Lichtkurve natürlich sehr empfindlich davon $a b$, unter welchem Winkel das System beobachtet wird. Beispielsweise ergeben sich große Unterschiede, je nachdem ob der Neutronenstern und die Akkretionsscheibe während einer Bahnperiode vom Begleitstern verdeckt werden 


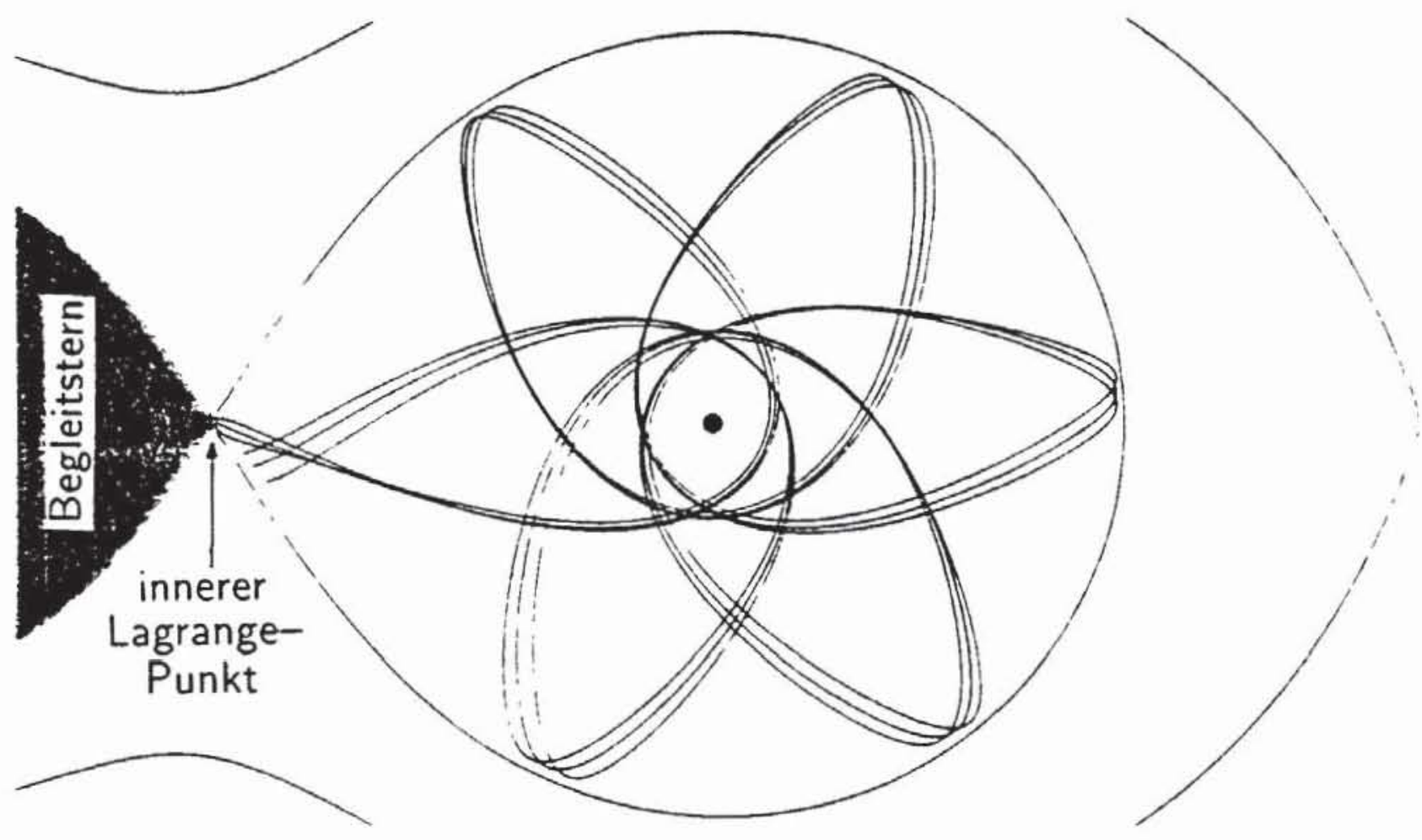

Abb. 3.2: Einteilchenbahnen im Roche-Potential mit etwas unterschiedlichen Anfangsbedingungen am inneren Lagrange-Punkt

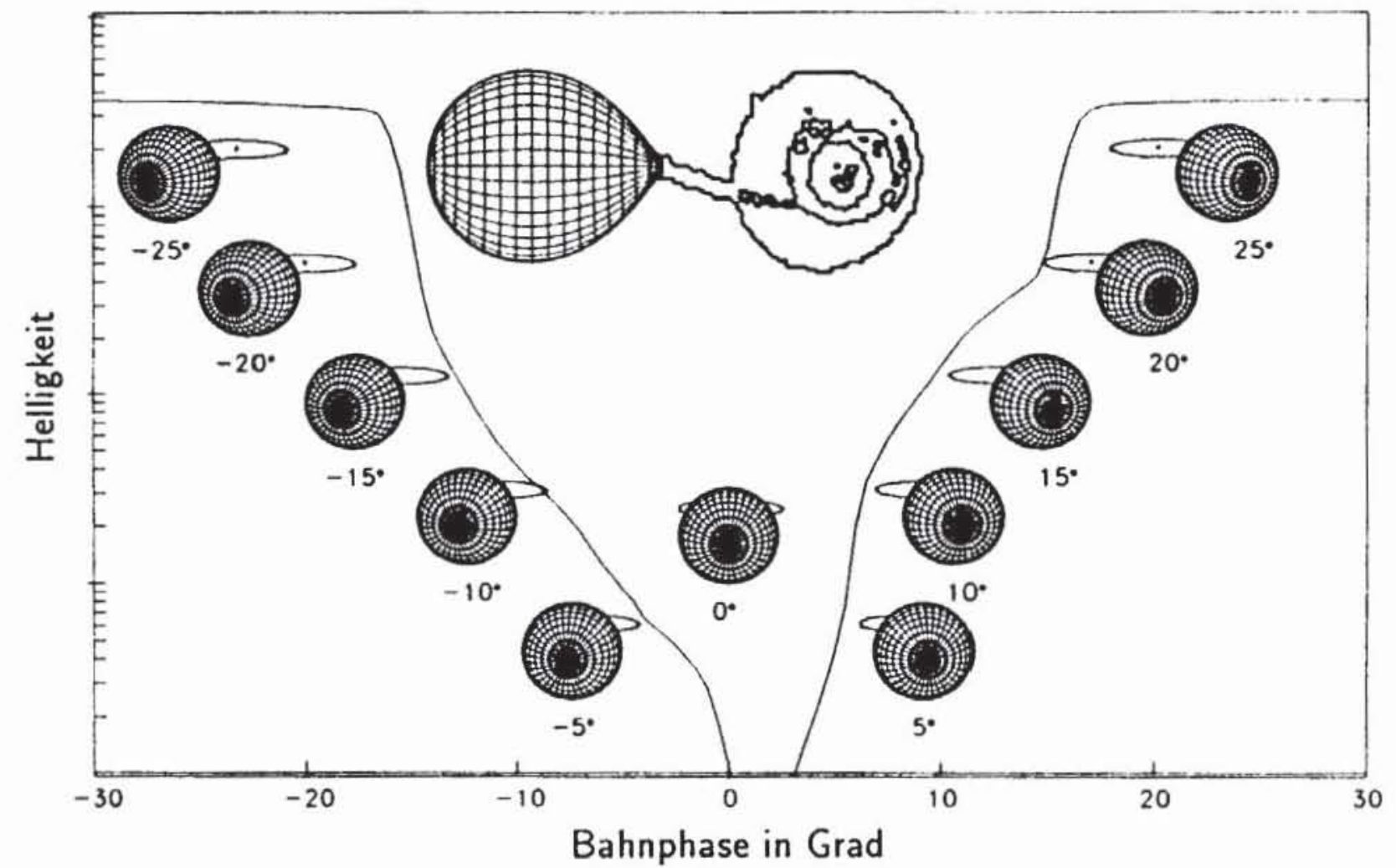

Abb. 3.3: Beispiel einer Lichtkurve für die Bedeckung einer Akkretionsscheibe. Die jeweilige Stellung des Systems relativ zum Beobachter ist in Schritten von 5 Grad eingezeichnet. Die Draufsicht zeigt die räumliche Struktur der Scheibe. 
oder nicht. Die Abb. 3.3 zeigt eine typische Lichtkurve für die Bedeckung einer Akkretionsscheibe. Zur Veranschaulichung ist auch noch die jeweilige Stellung des Systems relativ zum Beobachter gezeichnet.

Als besonders wichtig für unsere Untersuchungen von Akkretionsscheiben hat sich eine gute Diagnostik erwiesen. Eine Rechnung, beginnend mit der Bildung der Scheibe bis hin zu einem stationären Zustand mit etwa 20000 Teilchen, benötigt viele Cray 2 CPU-Stunden und erzeugt einige 100 MByte an Daten. Um die Vorgänge bei der Scheibenbildung, die Entstehung von Spiralstrukturen, die Ausbildung von Instabilitäten usw. verfolgen zu können, müssen diese Daten als Filmsequenzen aufbereitet werden. Abb. 3.4 zeigt eine Zeitserie für die Entwicklung einer Scheibe. Wir beginnen die Rechnungen ohne Scheibe und lassen kontinuierlich Materie vom normalen Stern auf den kompakten Stern überströmen. Nach vielen Umlaufperioden (typisch mehrere Stunden) hat die Scheibe ihren endgültigen Zustand erreicht. Dann strömt vom inneren Rand genau soviel Materie auf den kompakten Stern wie der Scheibe vom Begleitstern zugeführt wird.
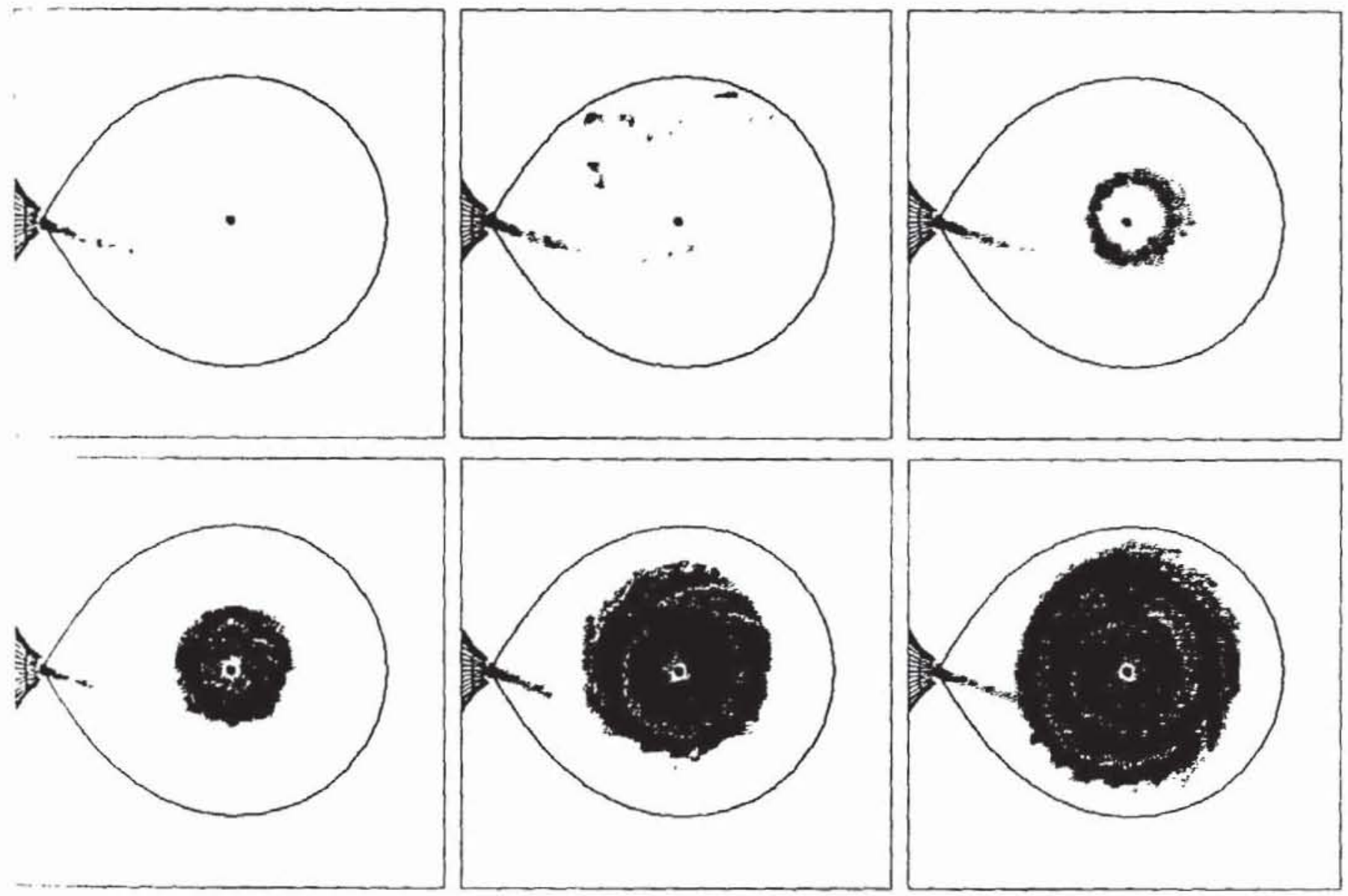

Abb. 3.4: Zeitserie der Entwicklung einer Akkretionsscheibe. Die Materie strömt vom normalen Stern durch die Gravitationswirkung auf den kompakten Stern und bildet dabei eine dünne Scheibe. Die Graustufen sind ein $\mathrm{MaB}$ für die Flächendichte.

Die Materie, die die Scheibe am inneren Rand verläßt, ist aufgrund der hohen Temperaturen ionisiert und muß daher den Magnetfeldlinien bis zu den Polen auf der Neutronensternoberfläche folgen. Der größte Teil der Röntgenstrahlung entsteht dort in der Abbremsregion, 
wo die Materie mit hoher Geschwindigkeit auftrifft. Die Abbremsung erfolgt innerhalb weniger Zentimeter. Die Strahlung muB dann durch den einfallenden Plasmastrom hindurch entweichen, bevor sie zum Beobachter gelangt. Die genaue Berechnung der Photonenausbreitung in diesem heißen, relativistisch schnell strömenden, stark magnetisierten Plasma ist ein außerordentlich schwieriges Problem. Die Plasmaströmung bewirkt eine Mitführung der Röntgenquanten, was zur Folge hat, daB diese die Säule bevorzugt in Richtung auf den Stern verlassen und so ringförmig um die Akkretionssäule auf die Neutronensternoberfläche auftreffen. Dort werden sie durch mehrfache Streuprozesse reflektiert, wodurch sich am Fuße der Säule ein im Röntgenlicht leuchtender Rıng bıldet.

\section{Lichtausbreitung in starken Gravitationsfeldern}

Ein Effekt, dessen Bedeutung für die korrekte Modellierung von physikalischen Phänomenen in der Umgebung stark gravitierender kompakter Objekte erst seit kurzem voll erkannt wurde, ist die Lichtablenkung in diesen starken Gravitationsfeldern. Licht wird, genauso wie jede andere elektromagnetische Strahlung auch, durch die gewaltige Gravitation - etwa 500 Milliarden mal größer als auf der Erdoberfläche - "angezogen"; seine Ausbreitung weicht von der geradlinigen Bahn ab. Während dieser Effekt bei der Sonne nur sehr gering ist (1.75 Bogensekunden am Sonnenrand), beträgt er in der Nähe eines Neutronensterns mehr als $45 \mathrm{Grad}$. Die Abb. 4.1 zeigt einige Photonenbahnen in der Schwarzschild-Metrik. Als eine Folge dieser Ablenkung kann auch Licht von Teilen der Rückseite des Sterns den Beobachter erreichen, man kann also teilweise um den Stern herumschauen, und es wird somit ein größerer Teil der Sternoberfläche sichtbar. Noch drastischer sınd dıe Effekte für Strahlung, die etwas (einige 100 Meter) oberhalb der Sternoberfläche entsteht. Diese Strahlung kann selbst dann zum Beobachter gelangen (s. Abb. 4.1), wenn das Emissionsgebiet genau hinter dem Stern liegt.

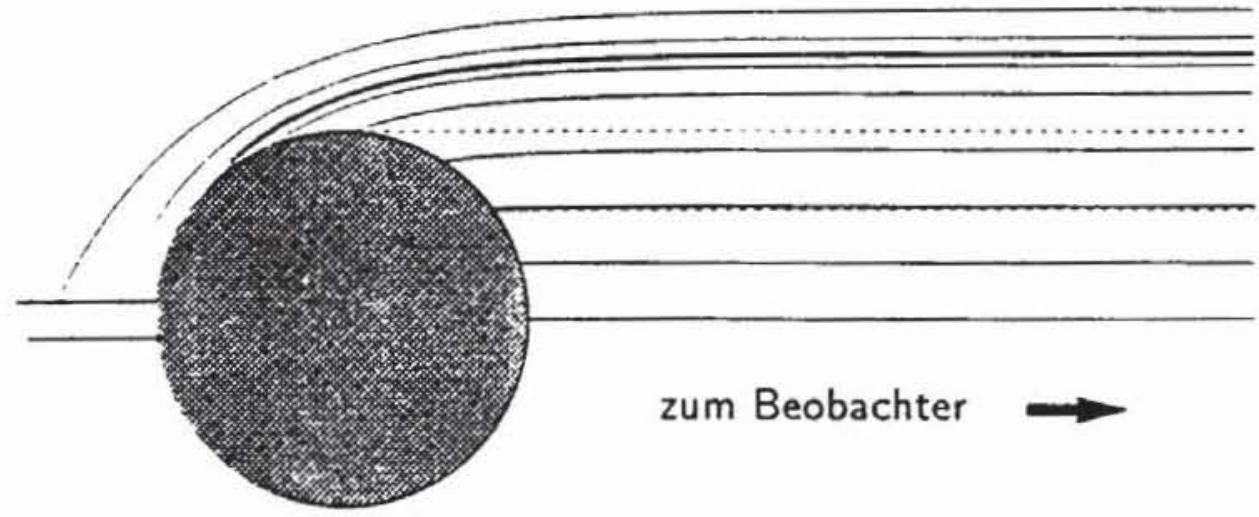

Abb. 4.1: Lichtablenkung bei einem Neutronenstern. Gezeichnet sind einige Bahnen von Photonen, die zu einem weit entfernten Beobachter gelangen.

Zur Verdeutlichung der Lichtablenkung und der dadurch bewirkten Effekte haben wir berechnet, wie für einen entfernten Beobachter ein Neutronenstern mit einem Ring (Abb. 4.2) und mit einem umlaufenden Begleitstern (Abb. 4.3) aussehen würde. Das dazu erforderliche Ray-Tracing in der Schwarzschild-Metrik ist relativ aufwendig, da die Bahn jedes einzelnen 
Photons durch numerische Integration bestimmt werden muß. Ein Bild mit einer Auflösung von $4800 \times 4800$ Punkten benötigt auf einer Cray 2 etwa 10 Minuten CPU-Zeit. Die hohe Auflösung ist zumindest lokal notwendig, da die Emission auf sehr kleinen Längen stark variieren kann.

Der wesentliche Unterschied zu der uns vertrauten Raum-Zeit-Struktur besteht darin, daß man durch die Lichtablenkung am Neutronenstern um ihn herumschauen kann. Dies hat zur Folge, daß man bei dem Ring in Abb. 4.2 gleichzeitig die Ober- und die Unterseite des hinter dem Neutronenstern liegenden Ringteils sieht. Hierin liegt der Grund für die verwirrende scheinbare Aufspaltung des Rings. Genau das gleiche gilt auch für den umlaufenden Stern (Abb. 4.3), von dem man Teile sowohl links als auch rechts vom Neutronenstern erkennt und der, wenn er sich hinter dem Neutronenstern befindet, sogar als ringförmiges Gebilde erscheint.

\section{Science Fiction}

Seit Albert Einstein vor 74 Jahren seine allgemeine Relativitätstheorie aufgestellt hat, gibt es ungezählte Geschichten und Filme, die versuchen, die diesen physikalischen Theorien zugrundeliegenden Raumzeitvorstellungen $\mathrm{zu}$ veranschaulichen. Da wir nicht täglich mit 99\% der Lichtgeschwindigkeit zu unserem Arbeitsplatz in der Nähe eines Schwarzen Loches fliegen, widerspricht die Raum-Zeit-Struktur der Relativitätstheorie vollkommen unserer tagtäglichen Erfahrung. Wir leben in unserer Vorstellung in einem dreidimensionalen euklidischen Raum, die Lichtgeschwindigkeit ist für uns praktisch unendlich. Daß dies bei Geschwindigkeiten, die nahe an der Lichtgeschwindigkeit liegen, nicht so ist, wird in der Relativitätstheorie beschrieben und ist experimentell mit hoher Genauigkeit bestätigt. Die dadurch auftretenden Erscheinungen - aus unserer Erfahrungswelt heraus als Paradoxa beurteilt - sind physikalische Realität.

Dank der Rechenlesstung moderner Supercomputer eröffnet sich hier ein reizvolles, weniger der Forschung, sondern mehr dem Verständnis dienendes Gebiet der Visualisierung der vierdimensionalen Raum-Zeit-Struktur der Relativitätstheorie. Die Grundidee ist einfach: man gibt sich physikalisch realistische Objekte vor, modelliert lokal ihr Emissionsverhalten, wie beispielsweise leuchtende Oberflächen, emittierende und absorbierende Volumina, und berechnet dann für einen ruhenden oder auf einer bestimmten Bahn fliegenden Beobachter mit Hilfe der Sehstrahlverfolgung in der gekrümmten Raumzeit Einzelbilder, aus denen sich Filmsequenzen aufbauen lassen.

Bewegt sich auch der Beobachter mit einer der Lichtgeschwindigkeit vergleichbaren Geschwindigkeit, sind überdies noch Lichtlaufzeiteffekte zu berücksichtigen, d.h. man muß rückwärts den Ort berechnen, an dem die Photonen emittiert wurden, die gleichzeitig ins Auge des Beobachters gelangen. Zusätzlich kann man diese Sequenzen für zwei Augenpositionen berechnen und so versuchen, sogar dreidimensionale Vorstellungen zu gewinnen. Eins ist jedoch schon sicher: Die Beschleunigung auf Warp-Geschwindigkeiten beim Raumschiff Enterprise oder im Krieg der Sterne sind zwar sehr phantasievoll gemacht, sie sind aber ebenso wie der Flug durch das Schwarze Loch in dem gleichnamigen Film - physikalisch falsch, so sähe es nicht aus! 

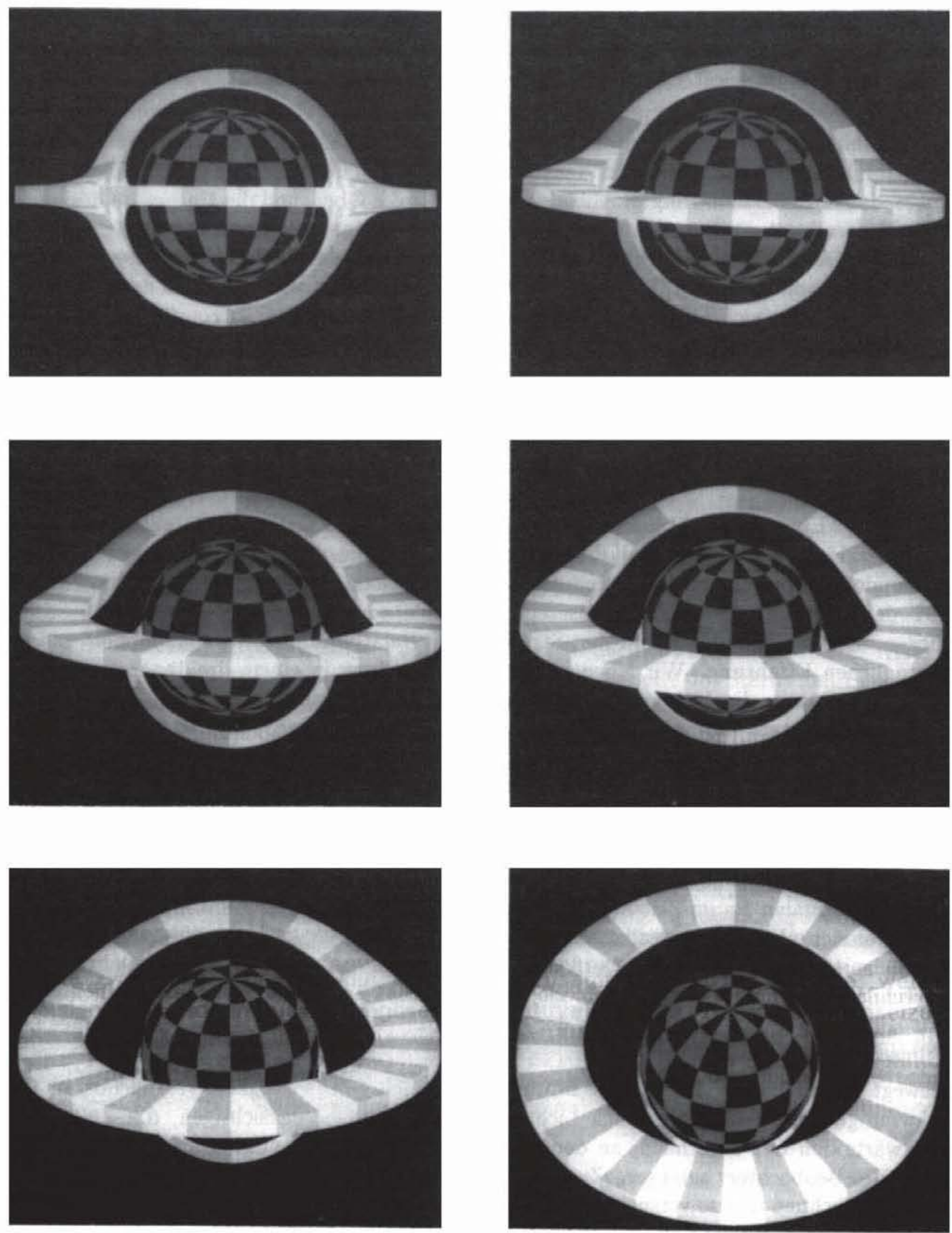

Abb. 4.2: Raum-Zeit-Struktur in der Umgebung eines Neutronensterns. Die Bilder zeigen einen zur besseren Veranschaulichung mit einem Karomuster überzogenen Neutronenstern und einen ihn umgebenden ebenfalls karierten Ring unter verschiedenen Blickwinkeln. Durch die gravitative Lichtablenkung ist gleichzeitig die Ober- und Unterseite des Rings zu sehen. Man beachte auch die gleichzeitige Sichtbarkeit der beiden Pole auf dem Neutronenstern. 

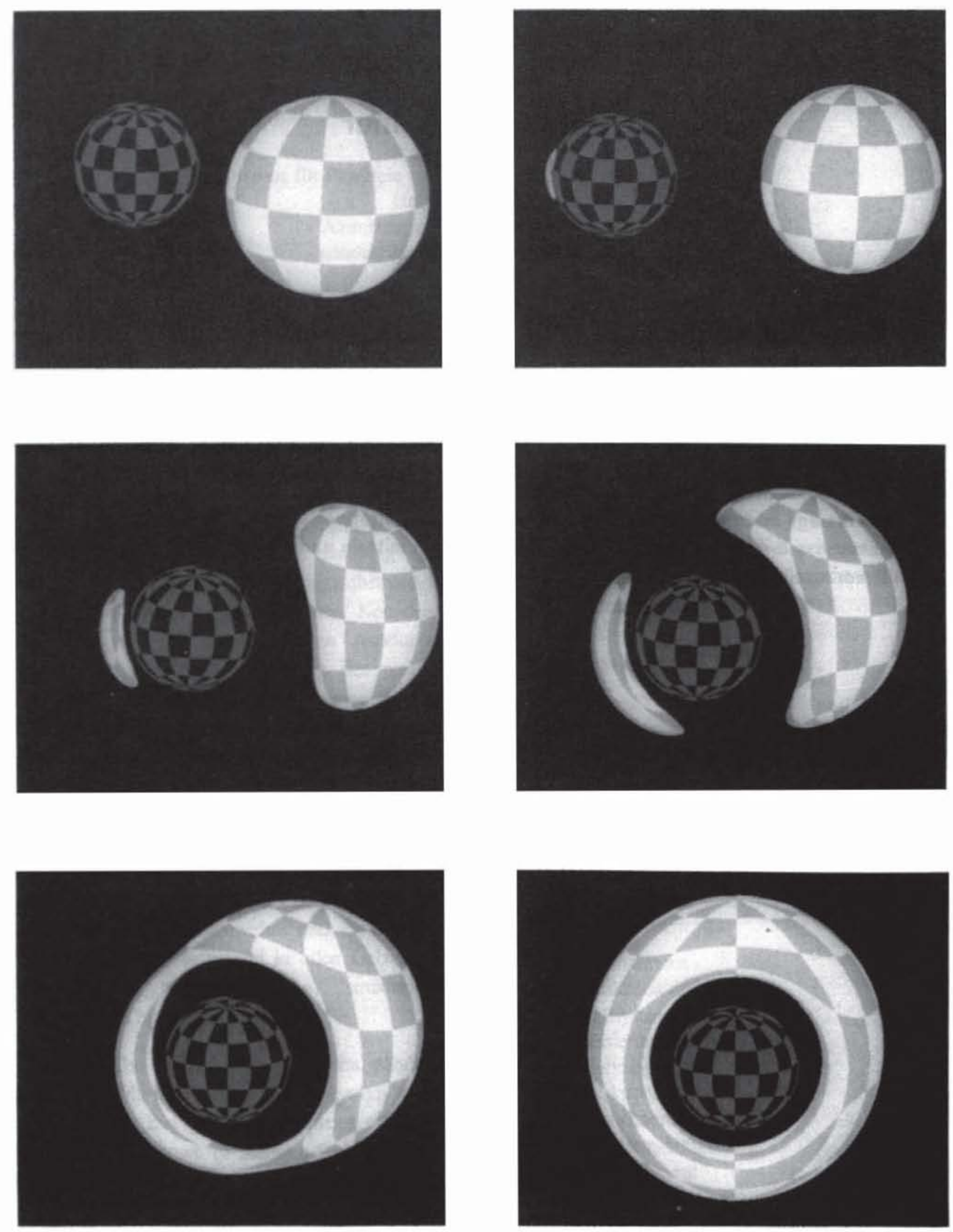

Abb. 4.3: Die Bilder zeigen sechs verschiedene Bahnphasen von dem Umlauf eines als masselos angenommenen Sterns um einen Neutronenstern. Durch die gravitative Lichtablenkung sieht man den Stern teilweise links und rechts vom Neutronenstern und, wenn er sich hinter dem Neutronenstern befindet, sogar als ein ringförmiges Gebilde. 